\title{
Confirmation of the factorial structure of the Japanese short version of the TEMPS-A in psychiatric patients and general adults
}

This article was published in the following Dove Press journal:

Neuropsychiatric Disease and Treatment

25 August 2016

Number of times this article has been viewed

\author{
Yasuya Nakato' \\ Takeshi Inoue ${ }^{1,2}$ \\ Shin Nakagawa' \\ Yuji Kitaichi' \\ Rie Kameyama' \\ Yumi Wakatsuki' \\ Kan Kitagawa' \\ Yuki Omiya' \\ Ichiro Kusumi' \\ 'Department of Psychiatry, Hokkaido \\ University Graduate School of \\ Medicine, Sapporo, ${ }^{2}$ Department of \\ Psychiatry, Tokyo Medical University, \\ Tokyo, Japan
}

Background: The Temperament Evaluation of the Memphis, Pisa, Paris, and San Diego Autoquestionnaire (TEMPS-A) is a 110-item questionnaire that assesses five affective temperaments. However, a valid shortened version is desired for large-scale investigations to enhance the compliance of respondents.

Methods: A confirmatory factor analysis was conducted among 320 psychiatric patients and 61 general adults. The participants completed the Japanese 39-item short version of the TEMPS-A, and a portion of the participants completed the 110-item version. An exploratory factor analysis with the principal factor method and varimax rotation was conducted to identify a more suitable model of the short version of the TEMPS-A.

Results: The confirmatory factor analysis revealed that the 39-item version exhibited a poor model fit. However, we found that the 18-item version exhibited a firm five-factor structure based on the exploratory factor analysis, and this model exhibited an acceptable model fit. It had good or acceptable internal consistency (Cronbach's $\alpha$ s: 0.672-0.819).

Limitations: The majority of the subjects in the present study were patients, and the temperament data may have been affected by psychiatric symptoms.

Conclusion: A firm five-factor structure was not found in the 39-item short version of the Japanese TEMPS-A. Therefore, an 18-item version was proposed. This new 18 -item version of the TEMPS-A might be useful for clinical applications and large-scale investigations.

Keywords: TEMPS-A, affective temperament, depression, validation, confirmatory factor analysis

\section{Introduction}

Mood disorders are subdivided into depressive and bipolar disorders. They are characterized by one or more mood episodes, depressive or (hypo)manic, and often show very long-term symptoms. In these disorders, symptoms cause clinically significant distress or impairment in social, occupational, or other important areas of functioning. ${ }^{1}$ Several affective temperaments have been considered to be antecedents or subsyndromal manifestations of mood disorders, particularly bipolar disorder. ${ }^{2}$

The items of the Temperament Evaluation of the Memphis, Pisa, Paris, and San Diego Auto-questionnaire (TEMPS-A) were formulated based on the diagnostic criteria for affective temperaments and were divided into the following five groups: cyclothymic, depressive, irritable, and hyperthymic groups, based on the subscales that represent the Kraepelinian temperaments, and anxious group, based on a subscale that was developed secondarily by Akiskal et $\mathrm{al}^{2-4}$ and Hantouche et $\mathrm{al}^{5}$ Affective temperaments have been reported to exert important pathoplastic actions on a wide range of diseases
Correspondence: Takeshi Inoue Department of Psychiatry, Tokyo Medical University, 6-7-I Nishishinjuku, Shinjuku-ku, Tokyo 160-0023, Japan

Tel +8I 3334261 II (ext 5754)

Fax +8I 333404499

Email tinoue@tokyo-med.ac.jp 
that extend far beyond the field of affective illness. ${ }^{6}$ Because the cyclothymic and hyperthymic temperaments predict drug responses to lithium and antidepressants, initial evaluations of affective temperaments may be useful for selecting treatment regimens for depressed patients. ${ }^{7}$ Although the original scale (110 items) of the TEMPS-A exhibited satisfactory results, the number of shortened versions that are more suitable for large-scale investigations and potentially enhance the compliance of respondents is increasing. ${ }^{4,8-13}$

The original 39-item short version of the TEMPS-A was extracted from the full English version and developed by Akiskal et al and translated into French, Italian, and Chinese. ${ }^{4,9,10,13}$ The subscales of the full and short versions of the TEMPS-A provide unique profiles of major depressive disorder and bipolar disorder and are very useful in clinical practice, particularly for bipolar spectrum diagnoses. ${ }^{7,14-16}$ Variants of the short version with different numbers of items have also been developed in other languages. ${ }^{8,11,12}$ The Japanese version of the TEMPS-A was translated and validated by Matsumoto et $\mathrm{al}^{14}$ and Akiyama et al. ${ }^{17}$. The 39-item Japanese short version has also been used clinically, but has not yet been validated. ${ }^{18}$

The five-factor structures of the English and Italian short versions of the TEMPS-A have been demonstrated by exploratory factor analysis (EFA) and confirmed by confirmatory factor analysis (CFA) in a few studies. ${ }^{4,10,19,20}$ In two CFA studies of the 39-item short TEMPS-A, the five-factor structure was confirmed, but the models did not exhibit good fits; among the goodness-of-fit indices, the root mean square errors of approximation (RMSEAs) were good, but the comparative fit indices (CFIs) were poor. ${ }^{19,20} \mathrm{CFA}$ is essential for testing the goodness of fit of the expected factor structure in clinical research involving questionnaires.

This study conducted CFA of the short version (39 items) of the TEMPS-A, which was translated from English to Japanese, to confirm the expected five-factor model with structural equation modeling. We proposed a new short version of the Japanese TEMPS-A, which exhibited a good fit to the model as determined by EFA using the principal factor method, varimax rotation, and subsequent CFA.

\section{Subjects and methods Subjects}

This research was conducted among 506 psychiatric patients and 120 general adults because the original short version of TEMPS-A was extracted in a mixed population of healthy controls and psychiatric patients. ${ }^{4}$ Among the 506 patients with mental disorders, 293 new outpatients and 213 inpatients of the Department of Psychiatry of Hokkaido University Hospital were consecutively included in the study from February 2008 to March 2011 and from April 2010 to November 2013, respectively. Complete responses to the questionnaires were provided by 158 of the 293 outpatients (53.2\%) and 162 of the 213 inpatients (76.1\%). Of the 120 general adult volunteers, 61 subjects $(50.8 \%)$ provided complete responses to the questionnaires. Overall, the investigated subjects included 320 psychiatric patients and 61 nonclinical general adults.

This study was performed according to the Declaration of Helsinki and was approved by the institutional review board of Hokkaido University Hospital. Written informed consent was obtained from all subjects.

\section{Psychiatric evaluations}

The Diagnostic and Statistical Manual of Mental Disorders, Fourth Edition, Text Revision (DSM-IV-TR) diagnoses of mental disorders in patients were made by trained psychiatrists using the Quick Reference to the Diagnostic Criteria from DSM-IV-TR, which contains the classification, diagnosis criteria, and a listing of the most important conditions to be considered in the differential diagnosis for each category; the demographic and clinical data were also recorded. The average interview duration was 60 minutes.

The nonclinical general adult volunteers completed a questionnaire to provide demographic and clinical data in addition to the TEMPS-A. None of these participants had previous histories of psychiatric disorders.

\section{Temperament Evaluation of the Memphis, Pisa, Paris, and San Diego Auto-questionnaire}

The TEMPS-A is a self-rated questionnaire consisting of 110 items (109 items for males, 110 items for females) that are rated as true $(=2)$ or false $(=1)$. It was designed to assess the following affective temperament dimensions: cyclothymic, depressive, irritable, hyperthymic, and anxious. ${ }^{2}$ Participants completed the Japanese 39-item version, which was extracted from the Japanese standardized full version (110 items) of the TEMPS-A based on the original English short version. ${ }^{4-14}$ The score for each temperament subscale is the mean score on the items that comprise that subscale.

The nonclinical general adults $(n=61)$ completed the 39-item short version of the TEMPS-A first and then completed the 110-item version of the TEMPS-A after an interval of at least 1 month.

\section{Statistical analysis}

According to the original 39-item English version of the TEMPS-A, we designed a structural equation model to perform the CFA (Figure 1). Each latent variable for each 


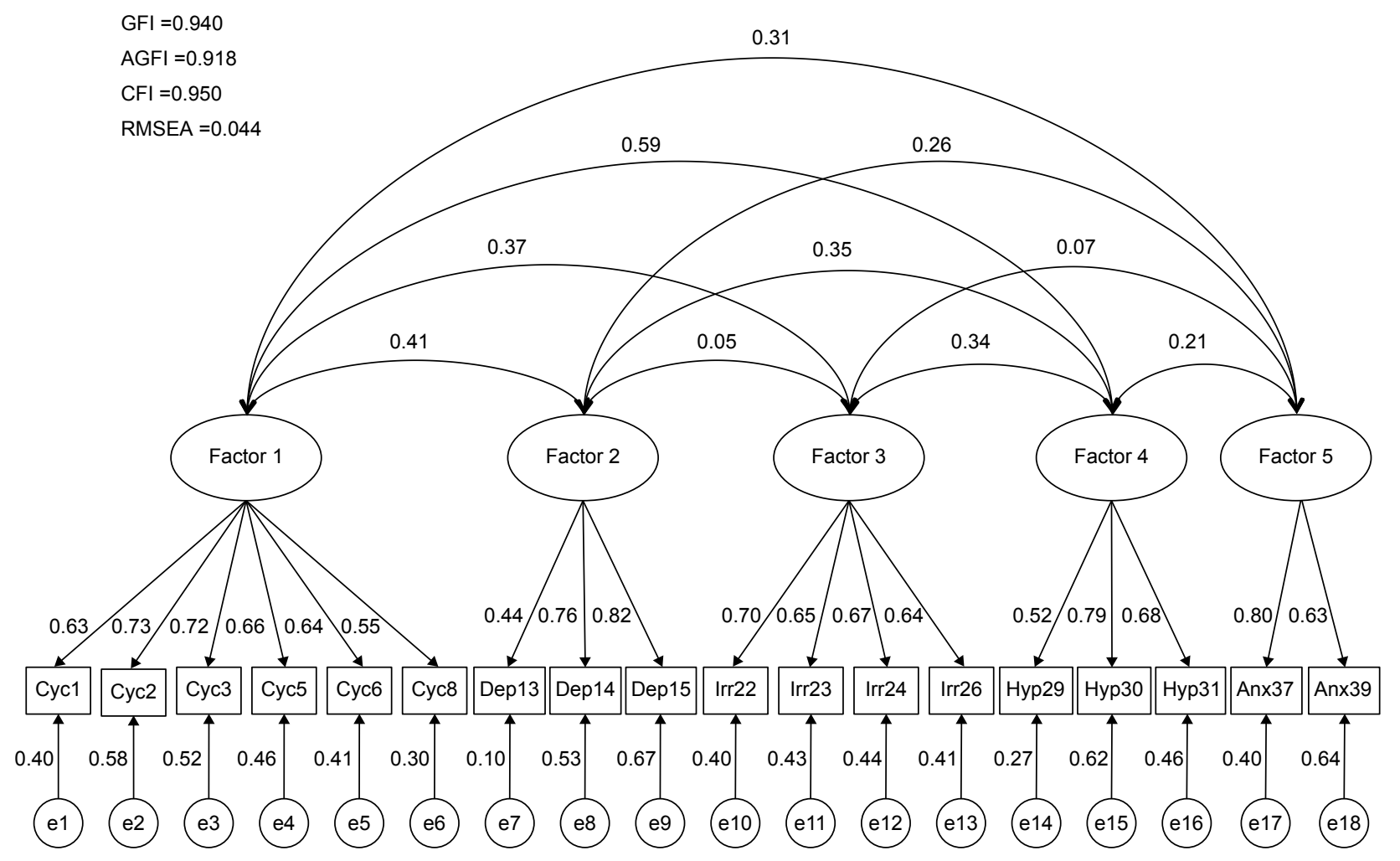

Figure I CFA of the 39-item TEMPS-A by structural equation modeling in mental disorder patients and general adults.

Abbreviations: CFA, confirmatory factor analysis; Cyc, cyclothymic; Dep, depressive; Hyp, hyperthymic; Anx, anxious; Irr, irritable; TEMPS-A, Temperament Evaluation of the Memphis, Pisa, Paris, and San Diego Auto-questionnaire; GFI, goodness-of-fit index; AGFI, adjusted goodness-of-fit index; CFI, comparative fit index; RMSEA, root mean square error of approximation.

temperament consisted of 3-12 observed variables. We used AMOS 20.0 (SPSS Inc., Chicago, IL, USA) to perform this CFA. We performed a maximum likelihood covariance estimation to analyze the model. For the inferential statistical evaluation of the structural equation modeling, we calculated the goodness-of-fit indices, which included the goodnessof-fit index (GFI), adjusted GFI (AGFI), CFI, and RMSEA. According to the conventional criteria, a GFI greater than 0.90, an AGFI greater than 0.85, a CFI greater than 0.95, and a RMSEA less than 0.08 indicated an acceptable fit, and a GFI greater than 0.95, an AGFI greater than 0.90, a CFI greater than 0.97 , and a RMSEA less than 0.05 indicated a good fit. ${ }^{21}$ It is recommended that several indices should be simultaneously evaluated because the indices represent different classes of goodness-of-fit criteria. ${ }^{21}$ We standardized and indicated all of the coefficients (to a maximum of 1 and a minimum of -1) for the covariance structure analysis.

A factor analysis with the principal factor method and varimax rotation was performed to evaluate the conceptual validity of the 39-item TEMPS-A and to perform the EFA.

The internal consistency of each subscale of the TEMPS-A was measured with Cronbach's $\alpha$.

Statistical analyses were performed using IBM SPSS for Windows 20 and IBM SPSS AMOS 20.0 (SPSS Inc.).
Differences were considered statistically significant at $P<0.05$.

\section{Results \\ Demographic and clinical data}

The demographic and clinical data of the subjects are shown in Table 1. Excluding the mood disorder patients, the patients' $(n=89)$ diagnoses consisted of nine cases of organic mood disorder, 13 cases of schizophrenia, two cases of a schizoaffective disorder, ten cases of a panic disorder, two cases of a social anxiety disorder, two cases of a pain disorder, two cases of an obsessive compulsive disorder, four cases of an eating disorder, ten cases of an adjustment disorder, eight cases of insomnia, and 27 other types of cases.

\section{Factorial structure of the Japanese short version of the TEMPS-A}

To evaluate the conceptual validity of the 39-item TEMPS-A, we performed a CFA with the five original subscales (ie, temperament scales) with AMOS 20.0. This model exhibited a poor model fit: $\mathrm{RMSEA}=0.052, \mathrm{GFI}=0.838$, AGFI $=0.818$, and $\mathrm{CFI}=0.809$ (Figure 1).

An EFA was then performed to examine the factor structure of the Japanese 39-item version of the TEMPS-A. 
Table I Demographic characteristics and the 39-item TEMPS-A data of subjects

\begin{tabular}{|c|c|c|c|c|c|}
\hline & \multicolumn{3}{|l|}{ Patients } & \multirow{2}{*}{$\begin{array}{l}\text { General adults } \\
(n=6 I)\end{array}$} & \multirow{2}{*}{$\begin{array}{l}\text { Total subjects } \\
(\mathrm{n}=\mathbf{3 8} \mathrm{I})\end{array}$} \\
\hline & BP $(n=92)$ & MDD (n=139) & Others $(n=89)$ & & \\
\hline Female & $48(52.2)$ & $79(56.8)$ & $48(53.9)$ & $10(16.4)$ & $185(48.6)$ \\
\hline Age (years) & $43.1 \pm 13.1$ & $44.4 \pm 16.9$ & $42.5 \pm 18.1$ & $40.1 \pm 10.9$ & $42.9 \pm 15.5$ \\
\hline \multicolumn{6}{|c|}{ Affective temperament scores of 39-item TEMPS-A } \\
\hline Cyclothymic & $1.46 \pm 0.28$ & $1.28 \pm 0.23 *$ & $1.37 \pm 0.30$ & $1.13 \pm 0.16$ & $1.32 \pm 0.27$ \\
\hline Depressive & $1.38 \pm 0.26$ & $1.36 \pm 0.28$ & $1.45 \pm 0.31$ & $1.16 \pm 0.21$ & $1.36 \pm 0.29$ \\
\hline Irritable & $1.26 \pm 0.26$ & $1.12 \pm 0.16^{\#}$ & $1.21 \pm 0.26$ & $1.11 \pm 0.15$ & $1.17 \pm 0.22$ \\
\hline Hyperthymic & $1.29 \pm 0.28^{\dagger}$ & $1.16 \pm 0.22$ & $1.20 \pm 0.23$ & $1.20 \pm 0.17$ & $1.21 \pm 0.23$ \\
\hline Anxious & $1.33 \pm 0.36$ & $1.37 \pm 0.36$ & $1.35 \pm 0.36$ & 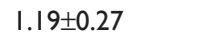 & $1.33 \pm 0.35$ \\
\hline
\end{tabular}

Notes: Data presented as mean \pm SD or numbers (percentages). *BP vs MDD, $P<0.000$ I; "BP vs MDD, $P<0.000$ I and MDD vs others, $P=0.0044$; ${ }^{+} B P$ vs $M D D, P=0.0002$ and $B P$ vs others, $P=0.0266$.

Abbreviations: TEMPS-A, Temperament Evaluation of the Memphis, Pisa, Paris, and San Diego Auto-questionnaire; BP, bipolar disorder; MDD, major depressive disorder; SD, standard deviation.

A factor analysis with the principal factor method and varimax rotation was performed with the five original subscales. The items were assigned to each subscale if they loaded on a specific factor at greater than 0.50 . The best model (Table 2), which consisted of 18 items, was extracted from the 39-item TEMPS-A. The first factor was defined by items $1,2,3,5,6$, and 8 and interpreted as the cyclothymic temperament. The second factor was defined by items 29, 30, and 31 and interpreted as the hyperthymic temperament. The third factor was defined by items 13, 14, and 15 and interpreted as the depressive temperament. The fourth factor was defined by items $22,23,24$, and 26 and interpreted as the irritable temperament. The fifth factor was defined by items 37 and 39 and interpreted as the anxious temperament.

Using the 18-item version of the TEMPS-A, which was statistically extracted as already described (Table 3), we performed a CFA with the five latent variables that corresponded to the temperament subscales. This model exhibited an acceptable model fit: RMSEA $=0.044$, GFI $=0.940$, AGFI $=0.918$, and CFI $=0.950$ (Figure 2).

Table 2 Principal factor analysis with varimax rotation of the 39-item TEMPS-A

\begin{tabular}{llll}
\hline & $\begin{array}{l}\text { Eigen } \\
\text { value }\end{array}$ & $\begin{array}{l}\text { Percentage } \\
\text { of variance } \\
\text { explained }\end{array}$ & $\begin{array}{l}\text { Cumulative } \\
\text { percentage }\end{array}$ \\
\hline Factor 1 & 3.825 & 9.808 & 9.808 \\
Factor 2 & 2.638 & 6.765 & 16.573 \\
Factor 3 & 2.619 & 6.715 & 23.287 \\
Factor 4 & 2.521 & 6.465 & 29.752 \\
Factor 5 & 1.433 & 3.673 & 33.426 \\
\hline
\end{tabular}

Note: All loadings and the explained variance by each factor.

Abbreviation: TEMPS-A, Temperament Evaluation of the Memphis, Pisa, Paris, and San Diego Auto-questionnaire.

\section{Internal consistency of the Japanese I8- item version of the TEMPS-A}

The internal consistencies of the temperament subscales (ie, cyclothymic, depressive, irritable, hyperthymic, and anxious) of the 18-item version of the TEMPS-A were examined. The Cronbach's $\alpha$ s were 0.819 for the cyclothymic, 0.697 for the depressive, 0.758 for the irritable, 0.692 for the hyperthymic, and 0.672 for the anxious temperament (Table 4). These values indicated that the Japanese 18-item version of the TEMPS-A exhibited good or acceptable internal consistency.

\section{Discussion}

The five-factor structure of the Japanese 39-item version of the TEMPS-A, which was extracted from the Japanese full version of the TEMPS-A, was investigated with CFA in the present study. However, due to the poor model fit, we were unable to confirm the expected five-factor structure of the 39-item version. Thus, we extracted 18 items from the 39-item version of the TEMPS-A by EFA. This 18-item version of the TEMPS-A exhibited favorable goodness-offit indices for the five-factor model. Thus, the results of this study indicate a limitation of the use of the 39-item Japanese version of the TEMPS-A in clinical research and practice. For these purposes, it is better to use the 18-item Japanese version of the TEMPS-A because this version has a firm five-factor structure.

The 110-item full version of the TEMPS-A has been translated into over 25 languages that have been used in clinical research and practice. ${ }^{722-29} \mathrm{~A}$ shorter version of the TEMPS-A is needed for research applications with large samples for severe patients because the 110-time version of the TEMPS-A requires a long time for completion. 
Table 3 Principal factor analysis with varimax rotation of the 39-item TEMPS-A

\begin{tabular}{|c|c|c|c|c|c|}
\hline Item & Factor I & Factor 2 & Factor 3 & Factor 4 & Factor 5 \\
\hline 2. I constantly switch between being lively and sluggish. & 0.671 & 0.156 & 0.061 & 0.119 & 0.085 \\
\hline 5. My mood often changes for no reason. & 0.620 & 0.018 & 0.219 & 0.261 & 0.075 \\
\hline 3. I get sudden shifts in mood and energy. & 0.607 & 0.142 & 0.091 & 0.196 & 0.083 \\
\hline I. My ability to think varies greatly from sharp to dull for no apparent reason. & 0.561 & 0.063 & 0.107 & 0.224 & 0.053 \\
\hline 8. I go back and forth between feeling overconfident and feeling unsure of myself. & 0.561 & 0.194 & 0.019 & 0.133 & 0.050 \\
\hline 6. I go back and forth between being outgoing and being withdrawn from others. & 0.515 & 0.180 & 0.164 & 0.203 & 0.038 \\
\hline 3I. I love to tackle new projects, even if risky. & 0.097 & $0.64 I$ & -0.105 & 0.120 & -0.043 \\
\hline 30. I often get many great ideas. & 0.190 & 0.605 & -0.039 & 0.126 & -0.016 \\
\hline 29. I have a gift for speech, convincing, and inspiring to others. & 0.104 & 0.567 & 0.073 & 0.071 & -0.009 \\
\hline I4. I am the kind of person who doubts everything. & 0.080 & 0.124 & 0.632 & 0.100 & 0.077 \\
\hline I5. I am a very skeptical person. & 0.144 & 0.032 & 0.626 & 0.163 & -0.013 \\
\hline 13. People tell me I am unable to see the lighter side of things. & 0.126 & -0.180 & 0.540 & 0.151 & 0.154 \\
\hline 22. I can get so furious that I could hurt someone. & 0.211 & 0.089 & 0.051 & 0.673 & 0.050 \\
\hline 26. When angry, I snap at people. & 0.170 & 0.130 & 0.121 & 0.612 & 0.066 \\
\hline 23. I often get so mad that I will just trash everything. & 0.279 & 0.019 & 0.184 & 0.566 & 0.054 \\
\hline 24. When crossed, I could get into a fight. & 0.215 & 0.167 & 0.086 & 0.550 & 0.039 \\
\hline 37. I am often fearful of someone in my family coming down with a serious disease. & 0.126 & 0.049 & 0.179 & 0.042 & 0.711 \\
\hline 39. When someone is late coming home, I fear they may have had an accident. & 0.061 & 0.091 & 0.062 & 0.087 & 0.648 \\
\hline
\end{tabular}

Notes: Loadings with values greater than 0.5 are shown. Item numbers indicate the item order on the English short version of the TEMPS-A. ${ }^{4}$ Abbreviation: TEMPS-A, Temperament Evaluation of the Memphis, Pisa, Paris, and San Diego Auto-questionnaire.

As noted in the introduction, the short 39-item version of the TEMPS-A has been developed and reported in only four languages. Although the French and Italian versions have been reported to exhibit five-factor structures based on CFA, the goodness-of-fit levels were good in terms of the RMSEAs but insufficient in terms of the CFIs, which were below 0.95 in two studies. ${ }^{19,20}$ The present study also found that the RMSEAs were sufficient, but the other three indices were insufficient for explaining this five-factor structure in the 39-item Japanese version of the TEMPS-A. It is

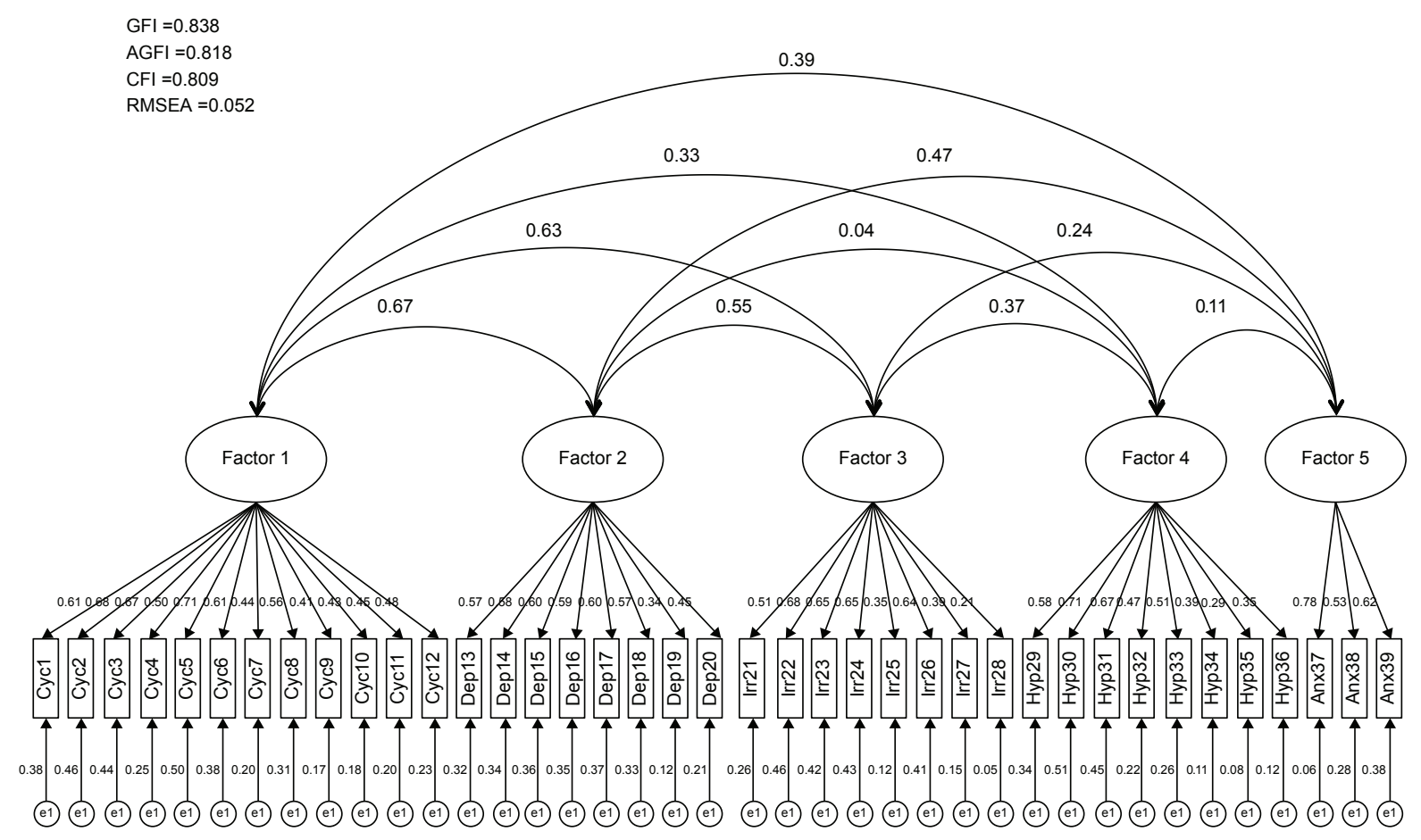

Figure 2 CFA by structural equation modeling of the 18-item TEMPS-A, which was extracted from the 39-item TEMPS-A by EFA in mental disorder patients and general adults.

Abbreviations: CFA, confirmatory factor analysis; Cyc, cyclothymic; Dep, depressive; Hyp, hyperthymic; Anx, anxious; Irr, irritable; TEMPS-A, Temperament Evaluation of the Memphis, Pisa, Paris, and San Diego Auto-questionnaire; EFA, exploratory factor analysis; GFI, goodness-of-fit index; AGFI, adjusted goodness-of-fit index; CFI, comparative fit index; RMSEA, root mean square error of approximation. 
Table 4 Internal consistency of temperament subscales of the I8-item TEMPS-A in all subjects $(\mathrm{N}=38 \mathrm{I})$

\begin{tabular}{lll}
\hline Temperament & Cronbach's $\alpha$ & Items \\
\hline Cyclothymic & 0.819 & $\mathrm{I}, 2,3,5,6,8$ \\
Depressive & 0.697 & $\mathrm{I} 3, \mathrm{I}, \mathrm{I}, \mathrm{5}$ \\
Irritable & 0.758 & $22,23,24,26$ \\
Hyperthymic & 0.692 & $29,30,3 \mathrm{I}$ \\
Anxious & 0.672 & 37,39 \\
\hline
\end{tabular}

Abbreviation: TEMPS-A, Temperament Evaluation of the Memphis, Pisa, Paris, and San Diego Auto-questionnaire.

recommended that several indices that represent different classes of goodness-of-fit criteria should be simultaneously evaluated. ${ }^{21}$ From this perspective, this model fits for the 39-item versions in three languages, including Japanese, were not good in terms of CFA.

By applying EFA to the Japanese short version of the 39-item TEMPS-A, we further extracted 18 items. CFA confirmed the five-factor structure of the 18-item TEMPS-A; all the four goodness-of-fit indices indicated acceptable or good fits. ${ }^{21}$ Therefore, at least regarding the Japanese short versions of the TEMPS-A, the 18-item version extracted from the 39-item version is recommended for clinical and research use. Because the smallest item number of the shortened version that had been previously reported was 35 in the German version, the smaller number of 18 items reported here is attractive and promising for clinical and research use. ${ }^{8}$

The limitation of this study is that the majority of the subjects of the present study were patients with mood disorders or other disorders, and the temperament data might have been affected by the psychiatric symptoms of these patients. In contrast, the majority of studies that have developed short versions of the TEMPS-A have been conducted on university students, whereas the original short version by Akiskal et al investigated mood disorder patients and healthy volunteers, similar to the present study. ${ }^{4,8,10,12}$ Measurement invariance across different age groups and across patients and healthy volunteers is important for a questionnaire in terms of the comparability of the results and should be confirmed. ${ }^{20}$

\section{Conclusion}

In conclusion, this study confirmed the firm five-factor structure of the 18-item but not the 39-item Japanese version of the TEMPS-A by CFA, indicating that the 18-item version is more suitable as a Japanese short version of the TEMPS-A than the 39-item version. Additional studies on the short TEMPS-A version should be performed using different age groups, and the five-factor structure should be confirmed by CFA in large populations that include patients and healthy subjects.

\section{Disclosure}

The authors report no conflicts of interest in this work.

\section{References}

1. American Psychiatric Association. Diagnostic and Statistical Manual of Mental Disorders, Fourth Edition, Text Revision (DSM-IV-TR). Washington, DC: American Psychiatric Publishing; 2000.

2. Akiskal HS, Akiskal KK, Haykal RF, et al. TEMPS-A: progress towards validation of a self-rated clinical version of the Temperament Evaluation of the Memphis, Pisa, Paris, and San Diego Autoquestionnaire. $J$ Affect Disord. 2005;85:3-16.

3. Akiskal HS. Toward a definition of generalized anxiety disorder as an anxious temperament type. Acta Psychiatr Scand. 1998;393:66-73.

4. Akiskal HS, Mendlowicz MV, Jean-Louis G, et al. TEMPS-A: validation of a short version of a self-rated instrument designed to measure variations in temperament. $J$ Affect Disord. 2005;85:45-52.

5. Hantouche EG, Akiskal HS. Toward a validation of a tripartite concept of a putative anxious temperament: psychometric data from a French national general medical practice study. J Affect Disord. 2005;85:37-43.

6. Rovai L, Maremmani AG, Rugani F, et al. Do Akiskal \& Mallya's affective temperaments belong to the domain of pathology or to that of normality? Eur Rev Med Pharmacol Sci. 2013;17:2065-2079.

7. Goto S, Terao T, Hoaki N, et al. Cyclothymic and hyperthymic temperaments may predict bipolarity in major depressive disorder: a supportive evidence for bipolar II1/2 and IV.J Affect Disord. 2011;129: 34-38.

8. Erfurth A, Gerlach AL, Hellweg I, et al. Studies on a German (Münster) version of the temperament auto-questionnaire TEMPS-A: construction and validation of the brief TEMPS-M. $J$ Affect Disord. 2005;85:53-69.

9. Krebs MO, Kazes M, Olie JP, et al. The French version of the validated short TEMPS-A: the temperament evaluation of Memphis, Pisa, Paris and San Diego. J Affect Disord. 2006;96:271-273.

10. Preti A, Vellante M, Zucca G, et al. The Italian version of the validated short TEMPS-A: the temperament evaluation of Memphis, Pisa, Paris and San Diego. J Affect Disord. 2010;120:207-212.

11. Ristic-Ignjatovic D, Hinic D, Bessonov D, et al. Towards validation of the short TEMPS-A in non-clinical adult population in Serbia. $J$ Affect Disord. 2014;164:43-49.

12. Woodruff E, Genaro LT, Landeira-Fernandez J, et al. Validation of the Brazilian brief version of the temperament auto-questionnaire TEMPS-A: the brief TEMPS-Rio de Janeiro. J Affect Disord. 2011; 134:65-76.

13. Yuan C, Huang J, Gao K, et al. Validation of the Chinese version of the short TEMPS-A and its application in patients with mood disorders. J Affect Disord. 2015;170:178-184.

14. Matsumoto S, Akiyama T, Tsuda H, et al. Reliability and validity of TEMPS-A in a Japanese non-clinical population: application to unipolar and bipolar depressives. J Affect Disord. 2005;85:85-92.

15. Mendlowicz MV, Akiskal HS, Kelsoe JR, et al. Temperament in the clinical differentiation of depressed bipolar and unipolar major depressive patients. $J$ Affect Disord. 2005;84:219-223.

16. Akiskal HS, Pinto O. The evolving bipolar spectrum: prototypes I, II, III and IV. Psychiatr Clin North Am. 1999;22:517-534.

17. Akiyama T, Tsuda H, Matsumoto S, et al. The proposed factor structure of temperament and personality in Japan: combining traits from TEMPS-A and MPT. $J$ Affect Disord. 2005;85:93-100.

18. Koda M, Kondo T. TEMPS-A/MPT as a quick finder for individualized treatments, including those targeting soft bipolarity. Clin Neuropsychopharmacol Ther. 2010;1:16-23.

19. Morvan Y, Tibaoui F, Bourdel MC, et al. Confirmation of the factorial structure of temperamental autoquestionnaire TEMPS-A in non-clinical young adults and relation to current state of anxiety, depression and to schizotypal traits. J Affect Disord. 2011;131:37-44.

20. Preti A, Vellante M, Gabbrielli M, et al. Confirmatory factor analysis and measurement invariance by gender, age and levels of psychological distress of the short TEMPS-A. J Affect Disord. 2013;151:995-1002. 
21. Schermelleh-Engel K, Moosbrugger H, Müller H. Evaluating the fit of structural equation models: tests of significance and descriptive goodness-of-fit measures. Methods Psychol Res Online. 2003;8:23-74.

22. Aguiar FA, Vasconcelos AG, Neves FS, et al. Affective temperaments: familiarity and clinical use in mood disorders. J Affect Disord. 2013;148:53-56.

23. Aguiar FA, Vasconcelos AG, Neves FS, et al. Affective temperaments and antidepressant response in the clinical management of mood disorders. J Affect Disord. 2014;155:138-141.

24. Iliceto P, Pompili M, Candilera G, et al. Suicide risk and psychopathology in immigrants: a multi-group confirmatory factor analysis. Soc Psychiatry Psychiatr Epidemiol. 2013;48:1105-1114.

25. Kesebir S, Vahip S, Akdeniz F, et al. Affective temperaments as measured by TEMPS-A in patients with bipolar I disorder and their first-degree relatives: a controlled study. J Affect Disord. 2005;85:127-133.
26. Pompili M, Rihmer Z, Akiskal H, et al. Temperaments mediate suicide risk and psychopathology among patients with bipolar disorders. Compr Psychiatry. 2012;53:280-285.

27. Pompili M, Innamorati M, Gonda X, et al. Affective temperaments and hopelessness as predictors of health and social functioning in mood disorder patients: a prospective follow-up study. J Affect Disord. 2013;150:216-222.

28. Pompili M, Innamorati M, Gonda X, et al. Characterization of patients with mood disorders for their prevalent temperament and level of hopelessness. J Affect Disord. 2014;166:285-291.

29. Rybakowski JK, Dembinska D, Kliwicki S, et al. TEMPS-A and longterm lithium response: positive correlation with hyperthymic temperament. J Affect Disord. 2013;145:187-189.
Neuropsychiatric Disease and Treatment

\section{Publish your work in this journal}

Neuropsychiatric Disease and Treatment is an international, peerreviewed journal of clinical therapeutics and pharmacology focusing on concise rapid reporting of clinical or pre-clinical studies on a range of neuropsychiatric and neurological disorders. This journa is indexed on PubMed Central, the 'PsycINFO' database and CAS,

\section{Dovepress}

and is the official journal of The International Neuropsychiatric Association (INA). The manuscript management system is completely online and includes a very quick and fair peer-review system, which is all easy to use. Visit http://www.dovepress.com/testimonials.php to read real quotes from published authors.

Submit your manuscript here: http://www.dovepress.com/neuropsychiatric-disease-and-treatment-journal 